\title{
Age-dependent changes in the protein expression levels of Redd1 and $m T O R$ in the gerbil hippocampus during normal aging
}

\author{
HEE-SOO CHOI ${ }^{1}$, JI HYEON AHN $^{2}$, JOON HA PARK ${ }^{3}$, MOO-HO WON $^{3}$ and CHOONG-HYUN LEE ${ }^{1}$ \\ ${ }^{1}$ Department of Pharmacy, College of Pharmacy, Dankook University, Cheonan, South Chungcheong 31116; \\ ${ }^{2}$ Department of Biomedical Science and Research Institute for Bioscience and Biotechnology, Hallym University, \\ Chuncheon, Gangwon 24252; ${ }^{3}$ Department of Neurobiology, School of Medicine, Kangwon National University, \\ Chuncheon, Gangwon 24341, Republic of Korea
}

Received March 2, 2015; Accepted December 30, 2015

DOI: $10.3892 / \mathrm{mmr} .2016 .4835$

\begin{abstract}
Redd1, also known as RTP801/Dig2/DDIT4, is a stress-induced protein and a negative regulator of mammalian target of rapamycin (mTOR). Redd1 is also closely associated with oxidative stress and DNA damage. In the present study, age-related changes in the protein expression levels of mTOR and Redd 1 were investigated using immunohistochemistry and western blot in the gerbil hippocampus at postnatal month (PM) 3, 6, 12 and 24. No significant differences were identified in the levels of mTOR among the experimental groups, whereas, the levels of phosphorylated mTOR decreased with age. The protein expression levels of Redd1 were observed to gradually increase with age; in the PM 24 group, the level was significantly increased ( 189.2\%), compared with the PM 3 group. In addition, Redd1 immunoreactivity was significantly increased in the hippocampal principal neurons of the PM 24 group, including the pyramidal cells in the hippocampus proper and granule cells in the dentate gyrus, compared with the other experimental groups. These results demonstrated that the protein expression of Redd1 in the hippocampus was markedly increased during normal aging, indicating that the age-related increase in the expression of Redd1 may be closely associated with age-related hippocampal change.
\end{abstract}

Correspondence to: Professor Moo-Ho Won, Department of Neurobiology, School of Medicine, Kangwon National University, 1 Kangwondaehak, Chuncheon, Gangwon 24341, Republic of Korea E-mail: mhwon@kangwon.ac.kr

Professor Choong-Hyun Lee, Department of Pharmacy, College of Pharmacy, Dankook University, 119 Dandae, Cheonan, South Chungcheong 31116, Republic of Korea

E-mail: anaphy@dankook.ac.kr

Key words: Redd1, mammalian target of rapamycin, hippocampus, aging, pyramidal cells, granule cells, gerbil

\section{Introduction}

Morphological and functional changes in the aged brain cause different types and degrees of neuropathological changes and neurobiological alterations (1-4). Aging of the brain leads to various changes, including oxidative stress, protein repair ability, DNA damage, autophagic degradation and neuronal activity in the aged brain (5-10). Among various brain regions, the hippocampus, which is important in the function of learning and memory, has been well established as a most vulnerable and susceptible region to the aging process $(11,12)$.

Mammalian target of rapamycin (mTOR) regulates cellular growth, proliferation and survival, and mediates a diverse range of cellular functions, including translation and transcription (13). It is understood that the inhibition of mTOR extends lifespan, whereas the overactivation of mTOR has been associated with the pathophysiology of age-related neurodegenerative diseases (14-16). In the hippocampus, the activation of mTOR is important for synaptic protein synthesis, dendrite arborization and excitatory synaptogenesis (17-19).

However, it has been widely accepted that Redd1 is a negative regulator of mTOR $(20,21)$. Redd1, which is also known as RTP801/Dig2/DDIT4, is a stress-induced protein expressed in neuronal and non-neuronal cells $(21,22)$. Redd1 is involved in the regulation of reactive oxygen species, and is regulated in response to hypoxia, oxidative stress, autophagy and cerebral ischemia (21-23). The induction of Redd 1 causes diverse functional consequences, including protecting cells from apoptosis induced by oxidative stress, as well as promoting post-mitotic neuronal death $(21,24)$. In addition, Redd1 has been investigated as an essential regulator of normal developmental neurogenesis and neuronal migration, as well as a direct regulator of mitochondrial metabolism (25-27).

A number of studies have shown age-related changes in the expression of mTOR in the hippocampus $(9,28)$. However, chronological changes in the expression of Redd1, as a negative regulator of mTOR, in the hippocampus during the process of normal aging remains to be fully elucidated. Therefore, in the present study, age-related changes in the 
protein expression levels of Redd1 were investigated in the hippocampus of the gerbil, which is considered to be a unique and suitable animal model for investigations on aging (29). This was performed in groups of animals at various ages using western blot and immunohistochemical analyses.

\section{Materials and methods}

Experimental animals. Male Mongolian gerbils (Meriones unguiculatus) were obtained from the Experimental Animal Center, Kangwon National University (Chuncheon, South Korea) at postnatal month (PM) 3, representing young animals, PM 6 and PM 12, representing adult animals, and PM 24, representing aged animals. The animals ( $n=14$ in each group) were housed in a conventional state under adequate temperature $\left(23^{\circ} \mathrm{C}\right)$ and humidity $(60 \%)$ control, with a 12 -h light/12-h dark cycle, and were provided with free access to food and water. The procedures for handling and caring for animals adhered to the guidelines in compliance with the Guide for the Care and Use of Laboratory Animals (30), and they were approved by the Institutional Animal Care and Use Committee at Kangwon National University (Chuncheon, South Korea; approval no. KW-130424-3). All experiments were performed in a manner to minimize the number of animals used and to minimize the suffering caused by the procedures used in the present study.

Western blot analysis. To examine changes in the protein levels of Redd1, mTOR and phosphorylated (p-)mTOR in the hippocampus between the adult and aged groups, animals in each age group $(n=7)$ were used, and western blot analysis was performed, according to the method used in our previous studies $(6,23,31)$. In brief, following sacrifice of the animals by cervical dislocation, the hippocampus was removed. The hippocampal tissues were homogenized in $50 \mathrm{mM}$ phosphate-buffered saline $\mathrm{PBS}$ ( $\mathrm{pH}$ 7.4) containing $0.1 \mathrm{mM}$ ethylene glycol bis (2 aminoethyl ether)-N,N,N',N' tetraacetic acid ( $\mathrm{pH} 8.0$ ), 0.2\% Nonidet $\mathrm{P}-40,10 \mathrm{mM}$ ethylendiamine tetraacetic acid ( $\mathrm{pH} 8.0), 15 \mathrm{mM}$ sodium pyrophosphate, $100 \mathrm{mM} \beta$-glycerophosphate, $50 \mathrm{mM} \mathrm{NaF}, 150 \mathrm{mM} \mathrm{NaCl}$, $2 \mathrm{mM}$ sodium orthovanadate, $1 \mathrm{mM}$ phenylmethylsulfonyl floride and $1 \mathrm{mM}$ dithiothreitol(DTT; all from Sigma-Aldrich). Following centrifugation at $16,000 \mathrm{x}$ g for $20 \mathrm{~min}$ at $4^{\circ} \mathrm{C}$, the protein levels were determined in the supernatants using a Micro BCA protein assay kit (Sigma-Aldrich), with bovine serum albumin as the standard (Pierce Biotechnology, Inc.; Thermo Fisher Scientific Inc., Waltham, MA, USA). Aliquots containing $20 \mu \mathrm{g}$ total protein were boiled in loading buffer containing $150 \mathrm{mM}$ Tris ( $\mathrm{pH}$ 6.8), 3 mM DTT, 6\% sodium dodecyl sulfate, $0.3 \%$ bromophenol blue and $30 \%$ glycerol (all from Sigma-Aldrich). The aliquots were then loaded onto a $10 \%$ polyacrylamide gel. Following electrophoresis, the gels were transferred onto nitrocellulose transfer membranes (Pall Life Sciences, East Hills, NY, USA). To reduce background staining, the membranes were incubated with $5 \%$ non-fat dry milk (Sigma-Aldrich) in PBS containing $0.1 \%$ Tween 20 (PBST; Sigma-Aldrich) for $45 \mathrm{~min}$ at room temperature. Following washing with PBS (x3), the membranes were incubated with polyclonal rabbit anti-human mTOR (cat. no. 2972; dilution, 1:1,000; Cell Signaling Technology, Inc., Danvers, MA, USA), polyclonal rabbit anti-human
phospho-mTOR (Ser2448; cat. no. 2971; dilution, 1:1,000; Cell Signaling Technology, Inc.), polyclonal rabbit anti-human Redd1 (cat. no. 10638-1-AP; dilution, 1:500; Proteintech, Chicago, IL, USA) or monoclonal mouse anti-human $\beta$-actin (cat. no. A5316; dilution, 1:5,000; Sigma-Aldrich) overnight at $4^{\circ} \mathrm{C}$. Following washing with PBST (x3), the membranes were incubated with peroxidase-conjugated donkey anti-rabbit IgG (cat. no. sc-2305) or goat anti-mouse IgG (cat. no. sc-2031; dilution, 1:1,000; Santa Cruz Biotechnology, Inc., Dallas, TX, USA) for $1 \mathrm{hr}$ at room temperature. An enhanced chemiluminescence kit (Pierce; Thermo Fisher Scientific, Inc.) was used to detect the protein expression. The results of the western blot analyses were scanned, and densitometric analysis for the quantification of the bands was performed using Image J 1.46 software (ImageJ, National Institutes of Health, Bethesda, MD, USA), which was used to determine the relative optical density (ROD). The levels of mTOR, p-mTOR and Redd1 were normalized with the level of $\beta$-actin. A ratio of the ROD was calibrated as the percentage, with the PM 3 group designated as $100 \%$.

Immunohistochemical detection of the expression of Reddl. For the immunohistochemical analyses, the animals $(n=7$ per group) were anesthetized with zoletil $50(30 \mathrm{mg} / \mathrm{kg}$; Virbac, Carros, France) and perfused transcardially with $0.1 \mathrm{M}$ phosphate-buffered saline (PBS; pH 7.4; Sigma-Aldrich), followed by $4 \%$ paraformaldehyde (Sigma-Aldrich) in $0.1 \mathrm{M}$ phosphate-buffer ( $\mathrm{pH}$ 7.4). The brain tissues were removed, cryoprotected and serially sectioned on a cryostat (CM1520; Leica Microsystems; Wetzlar, Germany) into $30 \mu \mathrm{m}$ coronal sections, and these were collected into six-well plates containing PBS.

According to the method used in our previous study $(6,23,31)$, immunohistochemical staining for Redd1 was performed using the polyclonal rabbit anti-human Redd1 primary antibody (1:200; cat. no. 10638-1-AP; Proteintech) a overnight at $4^{\circ} \mathrm{C}$. Following washing with PBS (x3), the brain tissues were incubated with biotinylated goat anti-rabbit IgG (BA-1000; 1:200; Vector Laboratories, Burlingame, CA, USA) for $2 \mathrm{hr}$ at room temperature and streptavidin peroxidase complex (SA-5004; 1:200; Vector Laboratories) for $45 \mathrm{~min}$ at room temperature. In order to establish the specificity of the immunostaining, a negative control assessment was performed using pre-immune serum (S-2012; 5\%; Vector Laboratories, Inc., Burlingame, CA, USA), which was used in place of the primary antibody. The negative control resulted in the absence of immunoreactivity in any structures (data not shown).

A total of seven sections, with $90-\mu \mathrm{m}$ intervals, per animal were selected to quantitatively analyze Redd1 immunoreactivity. Digital images of the hippocampal subregions were captured under an AxioM2 light microscope (Carl Zeiss AG, Jena, Germany) equipped with a digital camera (Axiocam; Carl Zeiss AG) connected to a PC monitor. Semi-quantification of the immunostaining intensities in the pyramidal cells of the hippocampus proper and in the granular cells of the dentate gyrus were evaluated using ImageJ 1.46 software (National Institutes of Health), according to the method of our previous study $(6,31)$. The mean intensity of the immunostaining in each of the immunoreactive structures was measured using a 0-255 gray scale system $(32,33)$, in which white to dark signal 
Table I. Mean intensity of Redd1 immunoreactivity in the principal cells of the gerbil hippocampus during normal aging.

\begin{tabular}{lccc}
\hline Cell type & PM 3 & PM 6 & PM 12 \\
\hline $\begin{array}{l}\text { Pyramidal cells of } \\
\text { hippocampus proper }\end{array}$ & + & ++ & ++ \\
$\begin{array}{l}\text { Granule cells } \\
\text { of dentate gyrus }\end{array}$ & \pm & + & +
\end{tabular}

Levels of immunoreactivity were defined into grades: Negative (-), weakly positive ( \pm ), moderate (+), strong (++) and very strong (+++). PM, postnatal month.

A
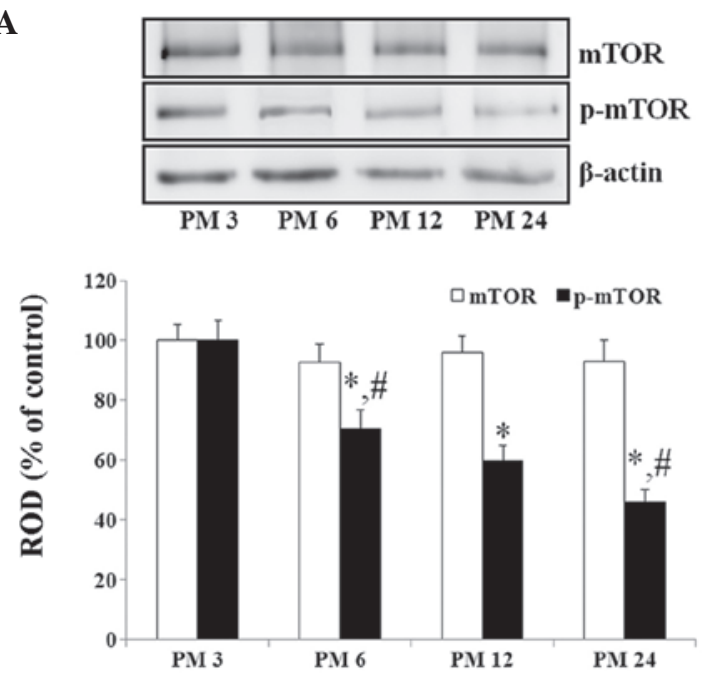

B
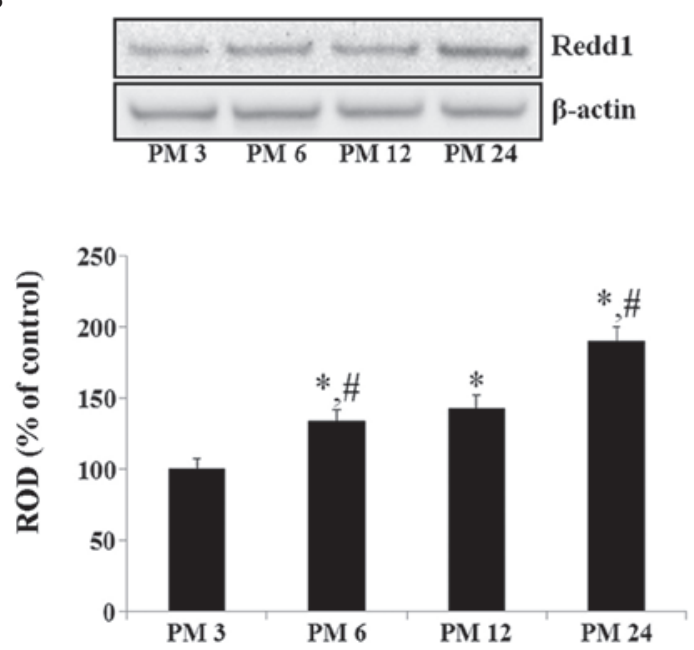

Figure 1. p-MTOR expression decreases and Redd1 expression increases with ageing. Western blot analysis of (A) mTOR, p-mTOR ( 289 kDa), and (B) Redd1 $(\sim 35 \mathrm{kDa})$ in the hippocampus, derived from gerbils of various ages. The ROD of the immunoblot bands is shown as a percentage of the control ( $n=7$ per group). ${ }^{*} \mathrm{P}<0.05$, compared with the PM 3 group; ${ }^{*} \mathrm{P}<0.05$, compared with the pre-time point group). Data are presented as the mean \pm standard error of the mean. mTOR, mammalian target of rapamycin; p-mTOR, phosphorylated mTOR; PM, postnatal month; ROD, relative optical density.
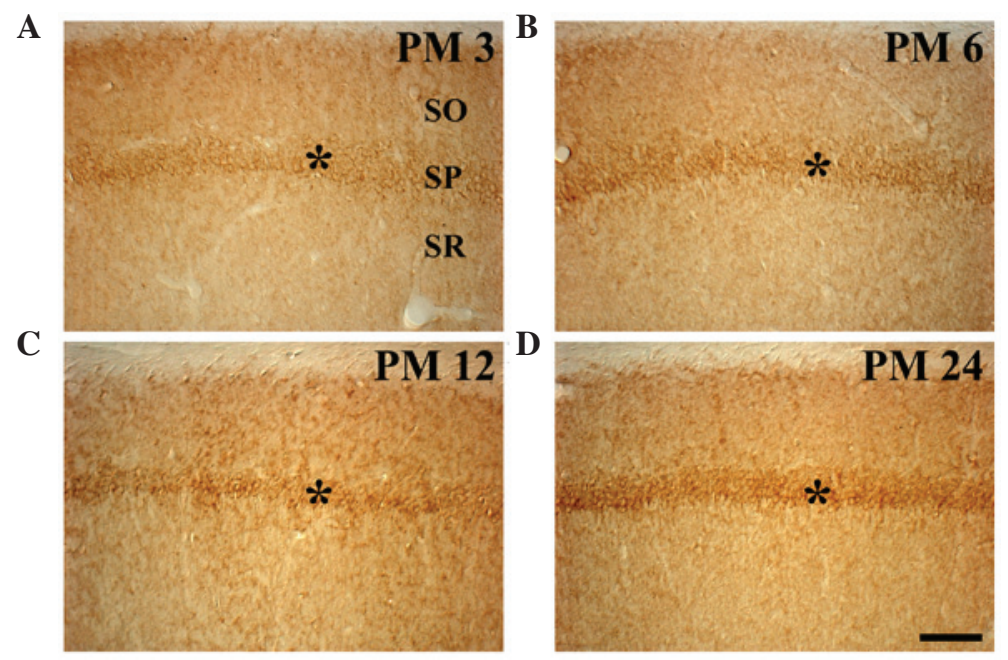

Figure 2. Immunohistochemical results for Redd1 in the hippocampal CA1 region at (A) PM 3, (B) PM 6, (C) PM 12 and (D) PM 24. Redd1 immunoreactivity increased in the SP $(*)$ of the PM 6 and PM 12 groups, compared with that in the PM 3 group. At PM 24, Redd1 immunoreactivity in the SP was markedly increased, compared with that in the PM 12 group. Scale bar $=100 \mu \mathrm{m}$. PM, postnatal month; SP, stratum pyramidale; SO, stratum oriens; SR, stratum radiatum.

corresponded to values between 255 and 0 , respectively). Based on this approach, the level of immunoreactivity was scaled as ,,,- \pm+++ or +++ , representing no staining (gray scale value: $\geq 200$ ), weakly positive (gray scale value: 150-199), moderate (gray scale value: 100-149), strong (gray scale value: 50-99) or very strong (gray scale value: $\leq 49$ ), respectively. 


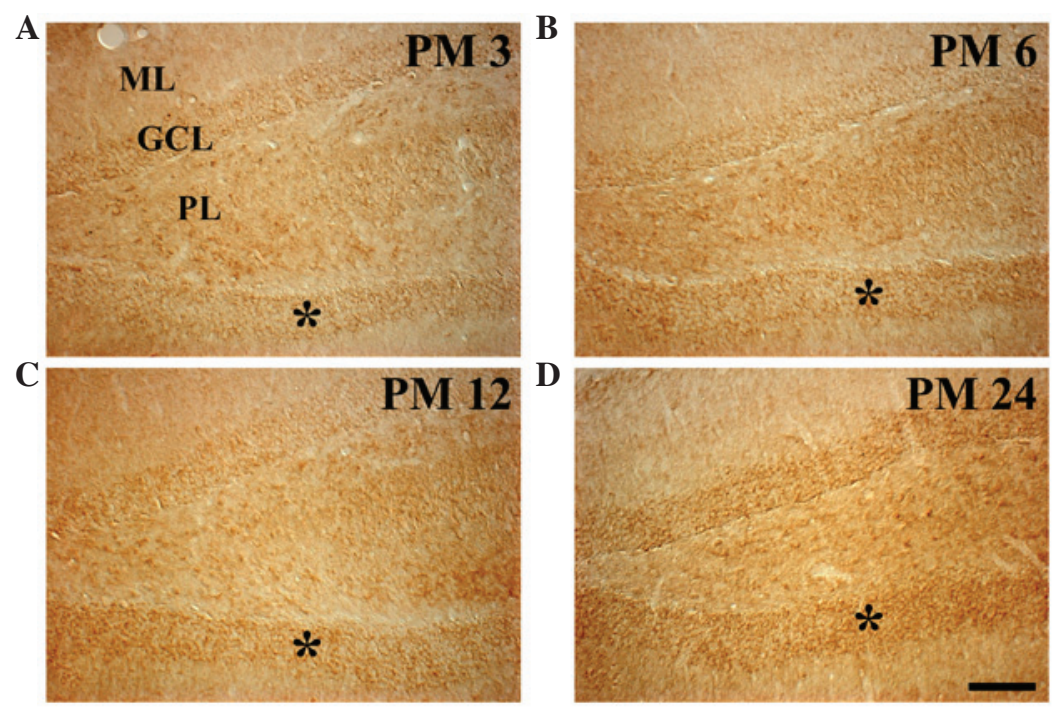

Figure 3. Immunohistochemical results for the expression of Redd1 in the dentate gyrus at (A) PM 3, (B) PM 6, (C) PM 12 and (D) PM 24. An age-dependent increase in Redd1 immunoreactivity was observed in the GCL (*) of the dentate gyrus. Scale bar=100 $\mu \mathrm{m}$. PM, postnatal month; GCL, granule cell layer; ML, molecular layer; PL, polymorphic layer.

Statistical analysis. Data are expressed as the mean \pm standard error of the mean. Differences between the mean ROD values among the groups were statistically analyzed using one-way analysis of variance, followed by Bonferroni's post-hoc multiple comparison test. $\mathrm{P}<0.05$ was considered to indicate a statistically significant difference.

\section{Results}

Changes in the protein levels of mTOR, p-mTOR and Redd1. From the results of the western blot analysis, no significant differences were observed in the levels of mTOR in the hippocampus among the experimental groups (Fig. 1A). However, the levels of p-mTOR in the hippocampus decreased in an age-dependent manner (Fig. 1A).

By contrast, the protein level of Redd1 in the hippocampus increased gradually during normal aging. The protein levels of Redd1 were significantly increased in the PM 6 and PM 12 groups (133.2 and $142.5 \%$, respectively), compared with the PM3 group. In addition, the protein level of Redd1 was markedly higher at PM 24, compared with the PM 6 and PM 12 group (132.7\% of the PM 12 group; Fig. 1B).

Changes in Redd1 immunoreactivity. In the PM 3 group, weak Redd1 immunoreactivity was observed in the pyramidal neurons of the stratum pyramidale (SP) of the hippocampus proper (CA1-3 regions; Table I; Fig. 2A). In particular, Redd1 immunoreactivity in the pyramidal neurons was detected in the cytoplasm, but not in the nucleus. In the PM 6 group, Redd1 immunoreactivity was increased; and moderate Redd1 immunoreactivity was observed in the SP of the hippocampus proper, compared with that in the PM 3 group (Table I; Fig. 2B). The Redd1 immunoreactivity in the hippocampus proper of the PM 12 group was similar to that observed in the PM 6 group (Table I; Fig. 2C). In the PM 24 group, Redd1 immunoreactivity in the SP of the hippocampus proper was markedly higher, compared with the PM 6 and PM 12 groups (Table I, Fig. 2D).
In the dentate gyrus of the PM 3 group, weak Redd1 immunoreactivity was observed in the granule cells of the granule cell layer (GCL) and in the polymorphic cells of the polymorphic layer (PL; Table I; Fig. 3A). The age-related change in Redd1 immunoreactivity in the GCL was similar to that observed in the hippocampus proper. In the GCL, the levels of Redd1 immunoreactivity were moderate in the PM 6 and PM 12 groups, and strong in the PM 24 group (Table I; Fig. 3B-D). However, no marked changes in Redd1 immunoreactivity were observed in the polymorphic cells of the PL among the experimental groups (Fig. 3).

\section{Discussion}

It is well established that mTOR is necessary for the normal development and viability of an organism, and that the inhibition of mTOR prolongs lifespan $(14,16,34)$. The inhibition of mTOR through rapamycin administration ameliorates age-related cognitive deficits, enhances learning and memory in young mice, and maintains memory in aged mice $(35,36)$. In addition, rapamycin treatment partially prevents behavioral decline in senescence-accelerated OXYS rats, which occurs by normalizing certain brain structures (37). In the present study, age-related changes were initially examined in the protein expression levels of mTOR and p-mTOR in the hippocampus, and an age-dependent decrease in the expression of p-mTOR Ser2448, but not mTOR, was found in the gerbil hippocampus. This result is in accordance with the results of previous studies, which demonstrated that the levels of p-mTOR Ser2448 decreased significantly in aged mice, compared with young mice $(9,28)$. Yang et al (9) showed that a decline in mTOR signaling activity was accompanied by a decline in brain-derived neurotrophic factor/phosphoinositide 3-kinase/Akt activity, and suggested that age-related dysfunction and diseases may be associated with the failure, rather than the overactivation, of mTOR decline as aging progresses. It was suggested that increased mTOR signaling in the hippocampus of the young mice 
may be due to it being more necessary for neuronal growth in young animals, than in adult and aged animals. In addition, it has been suggested that age-related cognitive decline might be related with a decrease of mTOR activation in the hippocampus (28).

In the present study, the protein level of Redd1 was also found to increase gradually during normal aging. In addition, it was found that Redd1 immunoreactivity increased gradually with age in the pyramidal and granule cells, which are known to be principal neurons in the hippocampus (38). To the best of our knowledge, the present study was the first study to demonstrate an age-dependent increase in the expression of Redd1 in the hippocampus. Although it is difficult to determine exactly why the expression of Redd1 was increased in the aged hippocampus, these finding may be supported by certain reports that showed that Redd1 was closely associated with oxidative stress and DNA damage, and that the accumulation of DNA damage and increase in oxidative stress were associated with the aging process in the brain $(5,8,22)$. In addition, Redd1 is a well-known negative regulator of mTOR $(20,21)$. Based on the previous and present findings, it was hypothesized that the increase of Redd1 in the aged hippocampus may be a compensatory mechanism against the elevation of DNA damage and oxidative stress, and that the enhanced expression of Redd1 may lead to a decline in mTOR activity.

In conclusion, the present study showed that the protein expression of Redd1 in the hippocampus was markedly increased with age during normal aging, and indicated that the age-dependent increase in Redd1 may be closely associated with age-related changes in the hippocampus, including an increase in DNA damage and oxidative stress, as well as changes in mTOR activity.

\section{Acknowledgements}

This study was supported by the Basic Science Research Program through the National Research Foundation of Korea, funded by the Ministry of Education (grant no. NRF-2014R1 A1A2058440), and by the Basic Science Research Program through the National Research Foundation of Korea, funded by the Ministry of Science, ICT and Future Planning (grant no. NRF-2014R1A2A2A01005307)'.

\section{References}

1. Hou XQ, Wu DW, Zhang CX, Yan R, Yang C, Rong CP Zhang L, Chang X, Su RY, Zhang SJ, et al: Bushen-Yizh formula ameliorates cognition deficits and attenuates oxidative stress-related neuronal apoptosis in scopolamine-induced senescence in mice. Int J Mol Med 34: 429-439, 2014.

2. Lister JP and Barnes CA: Neurobiological changes in the hippocampus during normative aging. Arch Neurol 66: 829-833, 2009.

3. Yang X, Wang JG, Ma DB, Ma XF, Zhu GJ, Zhou H, Yu CJ, Qian XY and Gao X: Myosin light chain kinase regulates hearing in mice by influencing the F-actin cytoskeleton of outer hair cells and cochleae. Int J Mol Med 33: 905-912, 2014.

4. Zou L, Yang R, Zhang P and Dai Y: The enhancement of amyloid precursor protein and beta-site amyloid cleavage enzyme 1 interaction: Amyloid-beta production with aging. Int J Mol Med 25 : 401-407, 2010.

5. Cardozo-Pelaez F, Brooks PJ, Stedeford T, Song S and Sanchez-Ramos J: DNA damage, repair, and antioxidant systems in brain regions: A correlative study. Free Radic Biol Med 28: $779-785,2000$
6. Lee CH, Park JH, Choi JH, Yoo KY, Ryu PD and Won MH: Heat shock protein 90 and its cochaperone, p23, are markedly increased in the aged gerbil hippocampus. Exp Gerontol 46: 768-772, 2011.

7. Rutten BP, Korr H, Steinbusch HW and Schmitz C: The aging brain: Less neurons could be better. Mech Ageing Dev 124 349-355, 2003

8. Shigenaga MK, Hagen TM and Ames BN: Oxidative damage and mitochondrial decay in aging. Proc Natl Acad Sci USA 91: 10771-10778, 1994

9. Yang F, Chu X, Yin M, Liu X, Yuan H, Niu Y and Fu L: MTOR and autophagy in normal brain aging and caloric restriction ameliorating age-related cognition deficits. Behav Brain Res 264: 82-90, 2014

10. Zhang R, Kadar T, Sirimanne E, MacGibbon A and Guan J: Age-related memory decline is associated with vascular and microglial degeneration in aged rats. Behav Brain Res 235: 210-217, 2012.

11. Jacobson L, Zhang R, Elliffe D, Chen KF, Mathai S, McCarthy D, Waldvogel $\mathrm{H}$ and Guan J: Correlation of cellular changes and spatial memory during aging in rats. Exp Gerontol 43: 929-938, 2008.

12. Rosenzweig ES and Barnes CA: Impact of aging on hippocampal function: Plasticity, network dynamics, and cognition. Prog Neurobiol 69: 143-179, 2003.

13. Laplante M and Sabatini DM: MTOR signaling in growth control and disease. Cell 149: 274-293, 2012.

14. Harrison DE, Strong R, Sharp ZD, Nelson JF, Astle CM, Flurkey K, Nadon NL, Wilkinson JE, Frenkel K, Carter CS, et al: Rapamycin fed late in life extends lifespan in genetically heterogeneous mice. Nature 460: 392-395, 2009.

15. Maiese K, Chong ZZ, Shang YC and Wang S: MTOR: On target for novel therapeutic strategies in the nervous system. Trends Mol Med 19: 51-60, 2013.

16. Selman C, Tullet JM, Wieser D, Irvine E, Lingard SJ, Choudhury AI, Claret M, Al-Qassab H, Carmignac D, Ramadani F, et al: Ribosomal protein S6 kinase 1 signaling regulates mammalian life span. Science 326: 140-144, 2009.

17. Jaworski J, Spangler S, Seeburg DP, Hoogenraad CC and Sheng M: Control of dendritic arborization by the phosphoinositide-3'-kina se-Akt-mammalian target of rapamycin pathway. J Neurosci 25 : 11300-11312, 2005.

18. Kumar V, Zhang MX, Swank MW, Kunz J and Wu GY: Regulation of dendritic morphogenesis by Ras-PI3K-Akt-mTOR and Ras-MAPK signaling pathways. J Neurosci 25: 11288-11299, 2005.

19. Lee CC, Huang CC and Hsu KS: Insulin promotes dendritic spine and synapse formation by the PI3K/Akt/mTOR and Rac1 signaling pathways. Neuropharmacology 61: 867-879, 2011.

20. Ellisen LW: Growth control under stress: MTOR regulation through the REDD1-TSC pathway. Cell Cycle 4: 1500-1502, 2005.

21. Shoshani T, Faerman A, Mett I, Zelin E, Tenne T, Gorodin S, Moshel Y, Elbaz S, Budanov A, Chajut A, et al: Identification of a novel hypoxia-inducible factor 1-responsive gene, RTP801, involved in apoptosis. Mol Cell Biol 22: 2283-2293, 2002.

22. Ellisen LW, Ramsayer KD, Johannessen CM, Yang A, Beppu H, Minda K, Oliner JD, McKeon F and Haber DA: REDD1, a developmentally regulated transcriptional target of p63 and p53, links p63 to regulation of reactive oxygen species. Mol Cell 10: 995-1005, 2002.

23. Lee CH, Park JH, Cho JH, Ahn JH, Yan BC, Lee JC, Shin MC, Cheon SH, Cho YS, Cho JH, et al: Changes and expressions of Redd1 in neurons and glial cells in the gerbil hippocampus proper following transient global cerebral ischemia. J Neurol Sci 344: 43-50, 2014

24. Malagelada C, Jin ZH and Greene LA: RTP801 is induced in Parkinson's disease and mediates neuron death by inhibiting Akt phosphorylation/activation. J Neurosci 28: 14363-14371, 2008.

25. Horak P, Crawford AR, Vadysirisack DD, Nash ZM, DeYoung MP, Sgroi D and Ellisen LW: Negative feedback control of HIF-1 through REDD1-regulated ROS suppresses tumorigenesis. Proc Natl Acad Sci USA 107: 4675-4680, 2010.

26. Malagelada C, López-Toledano MA, Willett RT, Jin ZH, Shelanski ML and Greene LA: RTP801/REDD1 regulates the timing of cortical neurogenesis and neuron migration. J Neurosci 31: 3186-3196, 2011 .

27. Mansfield KD, Guzy RD, Pan Y, Young RM, Cash TP, Schumacker PT and Simon MC: Mitochondrial dysfunction resulting from loss of cytochrome c impairs cellular oxygen sensing and hypoxic HIF-alpha activation. Cell Metab 1: 393-399, 2005 
28. Yang L, Zhang J, Zheng K, Shen H and Chen X: Long-term ginsenoside Rg1 supplementation improves age-related cognitive decline by promoting synaptic plasticity associated protein expression in C57BL/6J mice. J Gerontol A Biol Sci Med Sci 69: 282-294, 2014.

29. Cheal ML: The gerbil: A unique model for research on aging. Exp Aging Res 12: 3-21, 1986.

30. Institute of Laboratory Animal Research, Committee for the Update of the Guide for the Care and Use of Laboratory Animals and National Research Council: Guide For The Care And Use Of Laboratory Animals. 8th edition. National Academies Press Washington, DC, p220, 2011.

31. Lee $\mathrm{CH}$ and Won $\mathrm{MH}$ : Increased dynamin-1 and -2 protein expression in the aged gerbil hippocampus. Cell Mol Neurobiol 34: 791-796, 2014.

32. Lazic SE: Statistical evaluation of methods for quantifying gene expression by autoradiography in histological sections. BMC Neuroscience 10: 1-15, 2009.

33. Varghese F, Bukhari AB, Malhotra R and De A: IHC Profiler: an open source plugin for the quantitative evaluation and automated scoring of immunohistochemistry images of human tissue samples. PloS one 9: e96801, 2014.
34. Murakami M, Ichisaka T, Maeda M, Oshiro N, Hara K, Edenhofer F, Kiyama H, Yonezawa K and Yamanaka S: MTOR is essential for growth and proliferation in early mouse embryos and embryonic stem cells. Mol Cell Biol 24: 6710-6718, 2004.

35. Halloran J, Hussong SA, Burbank R, Podlutskaya N, Fischer KE, Sloane LB, Austad SN, Strong R, Richardson A, Hart MJ and Galvan V: Chronic inhibition of mammalian target of rapamycin by rapamycin modulates cognitive and non-cognitive components of behavior throughout lifespan in mice. Neuroscience 223: 102-113, 2012.

36. Majumder S, Caccamo A, Medina DX, Benavides AD, Javors MA, Kraig E, Strong R, Richardson A and Oddo S: Lifelong rapamycin administration ameliorates age-dependent cognitive deficits by reducing IL- $1 \beta$ and enhancing NMDA signaling. Aging Cell 11: 326-335, 2012.

37. Kolosova NG, Vitovtov AO, Muraleva NA, Akulov AE, Stefanova NA and Blagosklonny MV: Rapamycin suppresses brain aging in senescence-accelerated OXYS rats. Aging (Albany NY) 5: 474-484, 2013.

38. Jinno S and Kosaka T: Stereological estimation of numerical densities of glutamatergic principal neurons in the mouse hippocampus. Hippocampus 20: 829-840, 2010. 